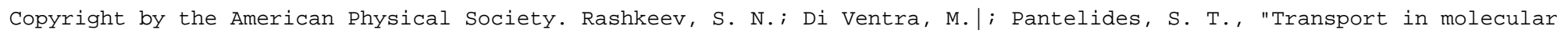

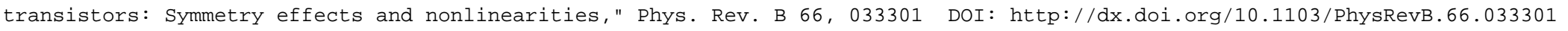

\title{
Transport in molecular transistors: Symmetry effects and nonlinearities
}

\author{
S. N. Rashkeev, ${ }^{1}$ M. Di Ventra, ${ }^{2}$ and S. T. Pantelides ${ }^{1,3}$ \\ ${ }^{1}$ Department of Physics and Astronomy, Vanderbilt University, Nashville, Tennessee 37235 \\ ${ }^{2}$ Department of Physics, Virginia Polytechnic Institute and State University, Blacksburg, Virginia 24061-0435 \\ ${ }^{3}$ Solid State Division, Oak Ridge National Laboratory, Oak Ridge, Tennessee 37831
}

(Received 29 April 2002; published 16 July 2002)

\begin{abstract}
We report first-principles calculations of the current-voltage and current-gate-field characteristics of model molecular transistors to explore the factors that control current amplification and other properties. We show that both the position and amplitude of resonant peaks are modified by the use of substituents that affect the symmetry and dipole moments of the molecules, and allow a linear versus nonlinear Stark effect. In addition, strong nonlinearities arise at large source-drain currents.
\end{abstract}

DOI: 10.1103/PhysRevB.66.033301

PACS number(s): 73.40.Jn, 73.40.Cg, 73.40.Gk, 85.65.+h

In recent years, the transport properties of single molecules have been extensively studied due to their potential use as active components of unconventional electronic devices. ${ }^{1}$ Some molecules can operate as diodes, ${ }^{2}$ Coulomb blockade structures, ${ }^{3}$ or switching devices with high negative differential resistance at room temperature. ${ }^{4,5}$ Transistorlike behavior in molecular three-terminal devices (molecular transistors) has been demonstrated in a single $\mathrm{C}_{60}$ fullerene connected to gold electrodes, ${ }^{6}$ in organic self-assembled monolayers, ${ }^{7}$ and in single- and multiwalled carbon nanotubes. 8 ,9 The source-drain current in these systems (three-terminal devices) is controlled by a gate voltage.

Theoretical investigations have so far elucidated the role of contact chemistry and geometry on the current-voltage characteristics of single molecules, ${ }^{10,11}$ the role of the injection energy at the contacts, ${ }^{12}$ stress-induced modification of transport properties, ${ }^{13}$ and the role of ligands for controlling temperature effects in molecular devices. ${ }^{14}$ Some transport properties of molecular devices (e.g., their temperature dependence) was also found to be intrinsically different from the properties of semiconductor nanostructures. 5,14

A molecular three-terminal device that could be an alternative to a metal-oxide-semiconductor transistor should exhibit characteristics such as amplification, signal-to-noise ratio, etc. Theoretical calculations for a benzene-1,4-dithiolate molecule with a third capacitive terminal (gate) have shown that some of these characteristics can, in principle, be realized. ${ }^{15}$ It was shown that the external gate field can significantly change the resistance of the molecule. ${ }^{15}$ When the gate field is swept through the resonance, the source-drain current changes by more than an order of magnitude. ${ }^{15}$ Such a device could be used to amplify the current at a fixed source-drain bias. The factors that control current amplification in molecular transistors remain an open question, however. Understanding these factors is necessary in the design of molecular devices with desired transport properties.

In this paper we explore the effect of symmetry on the current-voltage $(I-V)$ and the current-gate-field $(I-E)$ characteristics of model molecular transistors. In particular, we investigate the role of intrinsic dipole moments on the current modulation in these structures. The core molecules are constructed from the benzene-1,4-dithiolate molecule by substituting one or two hydrogen atoms by hydroxile groups
$(\mathrm{OH})$ (see Fig. 1). All molecules make contact with bulk electrode surfaces via sulfur atoms, and a third capacitative gate is applied perpendicular to the molecule plane (Fig. 1). The molecule with two $\mathrm{OH}$ groups has a twofold-symmetry axis perpendicular to the benzene ring while the molecule with one $\mathrm{OH}$ group does not. The transport properties of these two devices were found to be very different. The amplification of the current at the resonant tunneling regime is a few times larger in the asymmetric molecule, which is subject to the linear Stark effect in the gate electric field. At the same time, resonant tunneling occurs at much lower voltage than in the symmetric molecule. The shape of the $I-E$ characteristics depends on the value of the source-drain voltage in a nonlinear way, thus manifesting a strong dependence between the source-drain current and the gate electric field. Overall, the results illustrate that substituents and symmetry considerations can provide considerable control of the be-
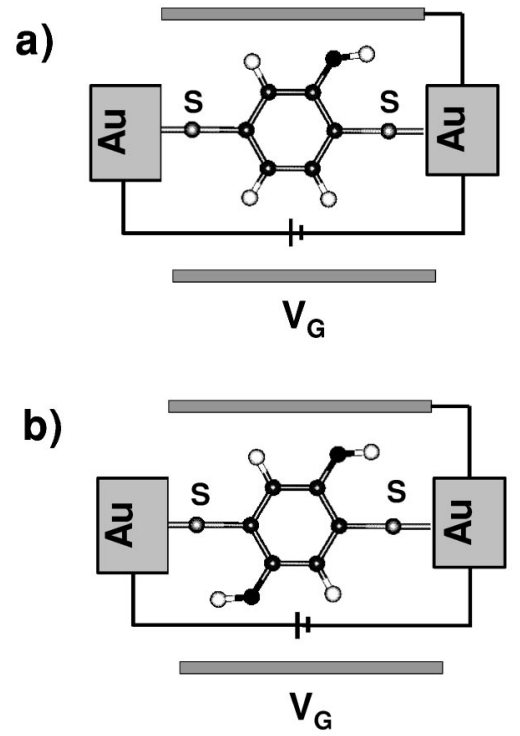

FIG. 1. The three-terminal geometry used in the present study. The molecules are derived from the benzene-1,4-dithiolate by replacing one (a) or two (b) hydrogen atoms by $\mathrm{OH}$ groups. The gate field lies in the plane of the benzene ring and perpendicular to the direction of electronic transport. Oxygen atoms are shown in black, hydrogen in white, carbon in gray. 
havior of molecular transistors.

We computed the $I-V$ characteristics of the molecular structures by using the method discussed in Ref. 16. The electron wave functions are computed by solving the Lippman-Schwinger equation iteratively to self-consistency in a steady state. ${ }^{16}$ Exchange and correlation are included in the density-functional formalism within the local-density approximation. ${ }^{17}$ The electrode surfaces are represented by an uniform positive background model (jellium model ${ }^{16}$ ) with the interior electron density of the electrodes equal to the value for metallic gold $\left(r_{s} \approx 3\right)$. The atomic geometry of the molecules was assumed to be the same as the one in vacuum without electrodes. The sulfur-surface distance was taken to be the one of sulfur adsorption on jellium surfaces. ${ }^{16}$ In the equilibrium configuration, the $\mathrm{C}-\mathrm{O}-\mathrm{H}$ bond angle is about $126^{\circ}$. Small variations in the bond distances do not affect the current considerably. ${ }^{16}$

The gate is introduced as a capacitor field generated by two circular charged disks kept at a fixed bias. ${ }^{15}$ The radius of the disks $(4.5 \AA)$ and the distance between them $(8 \AA)$ were chosen in such a way that the gate field covers the entire region of the molecule but does not affect the source and drain electrodes. The axis of the capacitor is perpendicular to the transport direction and lies in the plane of the benzene ring (Fig. 1).

The molecule shown in Fig. 1(a) has no inversion symmetry with respect to the gate field direction, while the molecule with two $\mathrm{OH}$ groups [Fig. 1(b)] has a twofoldsymmetry axis perpendicular to the benzene ring. We plot in Fig. 2 the density of states for the two molecules investigated. At zero gate field, the lowest-energy peaks (at about 5 $\mathrm{eV}$ below the Fermi level) of the density of states (DOS) correspond to the $\sigma$ orbitals of the benzene molecule with a small admixture from the $s$ orbitals of oxygen. The peaks around $-2.0 \mathrm{eV}$ correspond to a $\pi$-like orbital of the benzene ring. Their energies are closer to the Fermi level than the energy of the bonding $\pi$ orbital in a benzene-1,4dithiolate molecule ${ }^{10,14}$ due to the interaction of the $\pi$ orbital with the $\mathrm{OH}$-complexes $s p$ orbitals located at about $-4 \mathrm{eV}$. The calculated $I-V$ curves for both molecules are very similar despite the different symmetry (see Fig. 3). The similarity can be attributed to the fact that in both cases it is the bonding $\pi$ orbital that induces the peak in the current at about $2 \mathrm{~V}$.

When the gate field is applied, both molecules behave as resonant-tunneling transistors. Figure 4 shows the dependence of the current on the gate voltage for a source-drain voltage difference of $0.01 \mathrm{~V}$ for the two molecules of Fig. 1 and, as a reference, for the benzene-1,4-dithiolate molecule (see Ref. 15). All three molecules exhibit the same behavior: after a region of a constant high resistance the current increases, reaches a peak value, drops to a valley, and finally increases almost linearly with the gate field. In the case of the benzene-1,4-dithiolate molecule, the linear $I-E$ dependence at large gate fields is related to a participation of quasifree-electron states in resonant-tunneling processes, while the peak and the valley are due to resonant tunneling through $\pi^{*}$ antibonding states of the benzene ring. ${ }^{15}$ These states are

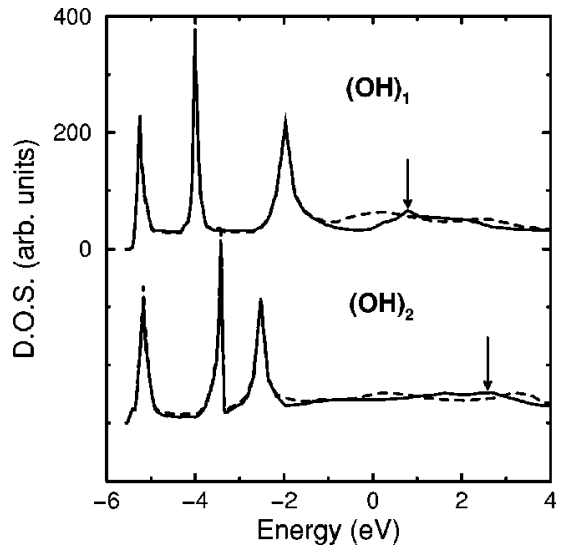

FIG. 2. Difference between the density of states of the two semi-infinite electrodes with and without the molecules of Fig. 1 in between, for a (source-drain) bias of $0.01 \mathrm{~V}$. The left Fermi level has been chosen as the zero of energy. Solid lines correspond to zero gate field, and dashed lines to the gate field $E=0.5 \mathrm{~V} / \AA$ for the asymmetric molecule, and to $E=1 \mathrm{~V} / \AA$ for the symmetric one, respectively. The vertical arrows indicate the position of the states responsible for resonant tunneling.

coupled to the continuum states when the gate field is applied and they experience a second-order Stark effect. ${ }^{15}$

An analysis of the DOS above the Fermi level for the two molecules of Fig. 1 shows that the shape of both $I-E$ curves is also determined by resonant-tunneling processes. For zero gate field and a bias voltage of $0.01 \mathrm{~V}$, the DOS of the first (asymmetric) molecule has a broad hump at $\sim 1$ eV (Fig. 2) due to antibonding $\mathrm{O}-\mathrm{H}$ orbitals hybridized with the $\pi$ state of the benzene ring. The states at about $\sim 1 \mathrm{eV}$ have a net dipole moment. Therefore the peak and the valley in the $I-E$ curve occur at lower gate fields in the case of the asymmetric molecule (see Fig. 4). On the other hand, the DOS curve for the second (symmetric) molecule is rather flat above the Fermi level with the antibonding $\pi^{*}$ state of the benzene ring at about $2.7 \mathrm{eV}$. As a result, the $I-E$ curve for the symmetric molecule is similar to that of the benzene-1,4dithiolate molecule. A field twice as large is needed to reach the resonant-tunneling condition for the $\pi^{*}$ antibonding states of the symmetric molecule (Fig. 4). We note also that the values of the current at the peak and at the valley (as well

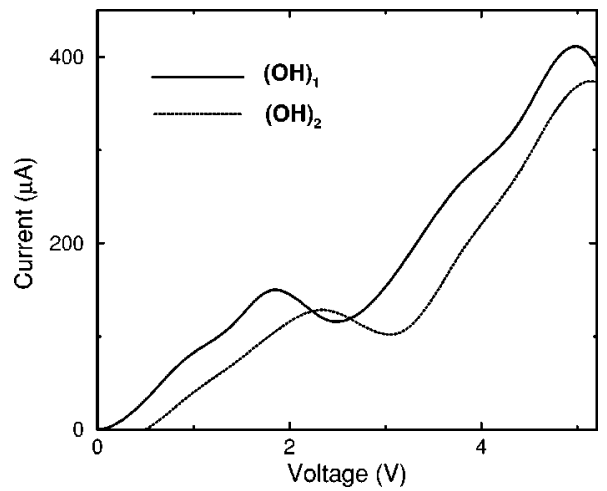

FIG. 3. Theoretical $I-V$ curves of the molecular structures of Fig. 1 for zero gate field. 


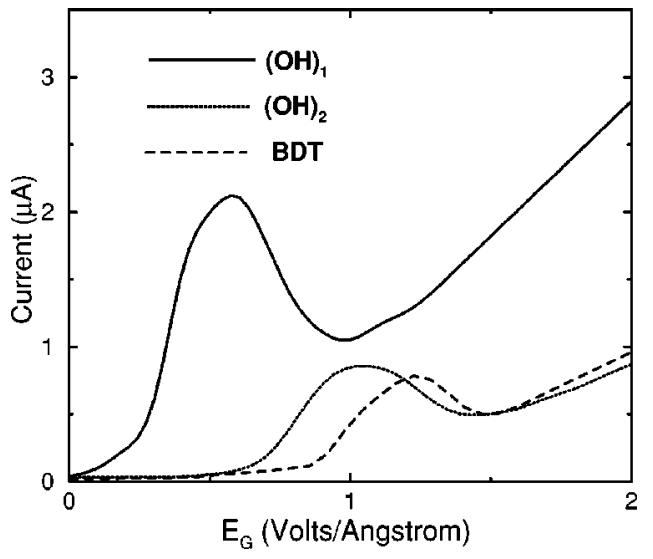

FIG. 4. Source-drain current as a function of the external gate field for the molecules of Fig. 1 and the benzene-1,4-dithiolate molecule (Ref. 15). The source-drain bias is $0.01 \mathrm{~V}$.

as the slope in the linear region) for the asymmetric molecule are two to three times larger than for the symmetric one. The reason for this difference can be attributed in this case to the larger DOS value at resonant tunneling for the molecule with a single $\mathrm{OH}$ group.

So far we described the I-E curves for a low value of the source-drain voltage $\left(V_{S-D}=0.01 \mathrm{~V}\right)$. However, the shape of the $I-E$ curve depends on the value of $V_{S-D}$ as well. Figure 5 shows the $I-E$ characteristics for both the considered molecules at a bias voltage of $2.4 \mathrm{~V}$. When the gate field is applied, the current in the two molecules changes in a different way. For the molecule with one $\mathrm{OH}$ group the current grows at low values of the gate field while for the molecule with two $\mathrm{OH}$ groups it drops. The reason for such a different behavior is clear from Fig. 3: for the molecule with two hydroxile groups the voltage $2.4 \mathrm{~V}$ corresponds to the voltage for which the $\pi$ states of the carbon ring are in resonance with the left Fermi level. The gate field couples these states with the states of the continuum, and the DOS peak shifts away from the left Fermi level. The resonant-tunneling con-

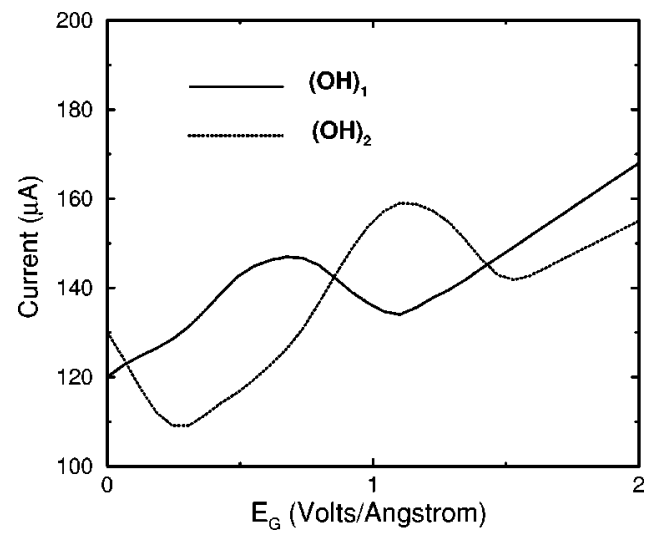

FIG. 5. Current in the two molecular devices of Fig. 1 as a function of the external gate field. The source-drain bias is $2.4 \mathrm{~V}$.

dition for this peak is not satisfied anymore, with consequent reduction in the current. Therefore, the external gate field can both increase or decrease the resistance of the device due to the nonlinear effects intrinsic in resonant tunneling. These results provide unique opportunities for current control in molecular devices.

In conclusion, we have shown that the symmetry of the core molecules in molecular transistors can significantly affect their transport properties. Also, the nonlinear interplay between the source-drain and gate fields can dramatically change the characteristics of molecular transistors. Both these results provide opportunities for the control of current in molecular structures.

This work was supported in part by the DARPA/ONR Grant No. N00014-99-1-0351, National Science Foundation Grant No. DMR-98-03768, the Oak Ridge National Laboratory, managed by UT-Battelle, LLC, for the U. S. Department of Energy under Contract No. DE-AC05-00OR22725, and by the William A. and Nancy F. McMinn Endowment at Vanderbilt University.

${ }^{10}$ M. Di Ventra, S.T. Pantelides, and N.D. Lang, Phys. Rev. Lett. 84, 979 (2000).

${ }^{11}$ S. Datta, W. Tian, S. Hong, R. Reifenberger, J.I. Henderson, and C.P. Kubiak, Phys. Rev. Lett. 79, 2530 (1997); M.P. Samanta, W. Tian, S. Datta, J.I. Henderson, and C.P. Kubiak, Phys. Rev. B 53, R7626 (1996); Y. Xue, S. Datta, and M.A. Ratner, J. Chem. Phys. 115, 4292 (2001); N.D. Lang and Ph. Avouris, Phys. Rev. B 64, 125323 (2001).

${ }^{12}$ S.N. Yaliraki, A.E. Roitberg, C. Gonzales, V. Mijica, and M.A. Ratner, J. Chem. Phys. 111, 6997 (1999).

${ }^{13}$ J.K. Gimzewski and C. Joachim, Science 283, 1683 (1999).

${ }^{14}$ M. Di Ventra, S.-G. Kim, S.T. Pantelides, and N.D. Lang, Phys. Rev. Lett. 86, 288 (2001).

${ }^{15}$ M. Di Ventra, S.T. Pantelides, and N.D. Lang, Appl. Phys. Lett. 76, 3448 (2000).

${ }^{16}$ N.D. Lang, Phys. Rev. B 52, 5335 (1995); M. Di Ventra and N. D. Lang, ibid. 65, 045402 (2002).

${ }^{17}$ P. Hohenberg and W. Kohn, Phys. Rev. 136, B864 (1964); W. Kohn and L.J. Sham, Phys. Rev. 140, A1133 (1965). 